\title{
Epidemiology of Pulmonary Aspergillosis
}

\author{
Dong-Na Su, Chen Qiu, Pu-Xuan Lu, and Shi-Ping Wu
}

Aspergillus exists extensively in the natural world that may be found in the soil, air, plants, wild or domestic fowl, and feathers of flying birds. It can also be commonly found at farms, stables, stalls, and barns. Aspergillus can parasite at human skin and upper respiratory tract but is not pathogenic due to human resistance to it. Aspergillosis is mostly secondary. When the immunity is compromised, the pathogenic aspergillus invades human lungs via the respiratory tract. It may also invade the skin and mucosa. In severe cases, septicemia occurs to involve other tissues and systems. Recent studies have shown that some strains of aspergillus may be carcinogenic. IgE-mediated allergic reaction may be triggered in anaphylactic person after inhalation of aspergillus spores to induce bronchial spasm.

Inhalation of aspergillus by individuals with normal immunity does not cause the disease. However, pulmonary aspergillosis occurs in immunocompromised individuals. It commonly occurs on the basis of diabetes, blood diseases, malignancies, large area burn, serious malnutrition, and other chronic wasting diseases. In other cases, pulmonary aspergillosis is induced due to long-term use of antibiotics, glucocorticoid, and/or immunosuppressant. In addition, it is commonly secondary to bronchiectasis, cavernous pulmonary tuberculosis, pulmonary cyst, pulmonary abscess, lung carcinoma, and other structural lung diseases.

In all the fungal infections following lung transplantation surgery, pulmonary aspergillosis accounts for about $44 \%$, and the incidence of pulmonary aspergillosis after parenchymal organ transplantation surgery is $21 \%$. It has been reported that the incidence of pulmonary aspergillosis after hematopoietic stem cell transplantation is $14-25 \%$ in China and $31 \%$ in the USA. The large-scale multicentered study in China directed by Prof. Liu [1] in the year 2011 has demonstrated that the lung fungal infections in patients with non-hematologic malignancies include pulmonary aspergillosis (180 cases, $37.9 \%$ ), pulmonary candidiasis (162 cases, $34.2 \%$ ), pulmonary cryptococcosis (74 cases, $15.6 \%$ ), pneumocystis (23 cases, $4.8 \%$ ), pulmonary mucormycosis (10 cases, $2.1 \%$ ), pulmonary penicilliosis marneffei (4 cases), and histoplasmosis (2 cases). However, there is no latest report about the incidence of pulmonary aspergillosis.

It is well known that Mycobacterium tuberculosis infection is the most common risk factor of chronic pulmonary aspergillosis across the world. A previous study in the UK have found that in patients with sputum Mycobacterium tuberculosis negative for 1 year after antituberculosis treatment, $14 \%$ was detected with sputum aspergillus and the aspergillus detection rate rose to $22 \%$ in patients with sputum Mycobacterium tuberculosis negative for 4 years after antituberculosis treatment. Another study found that the aspergillus antibody positive rate was $25 \%$ in patients with sputum Mycobacterium tuberculosis negative for 1 year. The data is possibly underestimated. Research indicated that the total case of chronic pulmonary aspergillosis secondary to tuberculosis may be up to 1.2 million. In some poorly equipped hospitals, chronic pulmonary aspergillosis may be misdiagnosed as complication of previous tuberculosis or relapse of tuberculosis because its presenting symptoms resemble to those of tuberculosis.

\section{Reference}

1. Liu Y, She D, Sun T, et al. Multicentered retrospective analysis of clinically defined cases of pneumomycosis during 1998 to 2007 in China. Chin J Tuberc Respir. 2011;34(2):86-90.

D.-N. Su · C. Qiu · S.-P. Wu $(\bowtie)$

Second Clinical Medical College of Jinan University,

Shenzhen, Guangdong, China

P.-X. Lu

Centre for Chronic Diseases Control of Shenzhen,

Shenzhen, Guangdong, China 